\title{
Objects of Concern: Caring for Children during Care Proceedings
}

\section{Chris Beckett and Bridget McKeigue}

Chris Beckett is a Principal Lecturer in Social Work at Anglia Ruskin University, Cambridge, and was previously the manager of a Children and Families social work team.

Bridget McKeigue is a Senior Lecturer in Social Work at Anglia Ruskin University, Cambridge, and was previously a child protection co-ordinator.

Correspondence to Chris Beckett, Webb Building, Anglia Ruskin University, East Road, Cambridge CB1 1PT, UK. E-mail: Chris.Beckett@anglia.ac.uk

This is a pre-copy-editing, author-produced PDF of an article accepted for publication in British Journal of Social Work following peer review. The definitive publisher-authenticated version Becket, C., \& McKeigue, B., 2009. Objects of concern: Caring for children during care proceedings. British Journal of Social Work, 2010, 40(7),pp.2086-2101 is available online at: http://bjsw.oxfordjournals.org/content/40/7/2086.abstract

This article is the second to look at the fifty-nine children who were made subject to care proceedings in one local authority area in the financial year 2004-05. It focuses on what happened to children during the proceedings and considers how well those responsible for their care during this time (the local authority and others involved in the court process) performed in terms of providing the children with parental care during the process. While the local authority in question performed well in relative terms, nevertheless, in respect of the duration of the process and the number of placement moves to which children were subjected, many of the children were subjected to a great deal of bewildering change, complex routines and large numbers of adults going in and out of their lives. The authors suggest that the process will tend to objectify children if it becomes too focused on decision making for the future, at the expense of thinking about their needs in the here and now. We conclude with some suggestions as to possible ways of improving things.

Keywords: Care proceedings, looked after children, child-care planning, court delay

\section{Introduction}

This is the second article to report on a study of all the care proceedings initiated by one local authority ('Local Authority X') over a one-year period. (For international readers: care proceedings, in England and Wales, are court proceedings that may result in the making of a 'care order', which allows a local authority to determine where a child is to live.) In the previous article (McKeigue and Beckett, 2009), we raised some questions about the precourt' period: the period before cases of this kind actually arrive in the court arena. We intend in a later article to discuss the 'post-court' period, after proceedings are over. However, here, we look at what happens during the 'in-court' period itself, the period between the initial and final hearings.

It is easy to think of the court process as simply a decision-making exercise, and the child during the in-court stage as waiting for a decision to be made, but, in our view, this is a dangerously one-dimensional way of seeing it. For a child, these proceedings (which typically last about eight to ten months and quite often well over a year) are not simply a decisionmaking moment. They are, in themselves, part of his or her childhood and will form a significant part of his or her entire experience of being cared for. Our fear is that if this is not taken seriously, the in-court period-inevitably a difficult time-could become a really traumatic episode in a child's life.

The case of 'Michael', from a previous study in another area (Beckett and McKeigue, 2003), will perhaps help to clarify our concerns. Michael was just under two when he was removed from home on an Emergency Protection Order (EPO). A full care order was not made on him until over two years later, when he was four years and four months old. However, it was another year and a half before he was placed with an adoptive family. He went through 
eleven placement changes between the first EPO and adoption. If we disregard short respite breaks, this figure reduces to seven changes involving five sets of carers. During the proceedings, he was involved in parenting skills assessments at two different family centres, he was seen by a psychiatrist and was involved in six sessions with a social worker from an adoption team to explore his understanding and wishes, and he had three different allocated social workers. His experience included him being placed in the care of his father and his father's partner on a trial basis, this arrangement breaking down, and then again being placed with his father and his father's partner some ten months later, only for this also to break down in the same way.

Our submission is that this experience, in itself, regardless of what happened to Michael before or afterwards, could very likely have caused him long-term psychological harm. And we suggest that this example demonstrates the need for adults involved in making decisions for children during the in-court period to take responsibility not only for making plans for the future, but also for ensuring that the children are properly cared for in the present. Our question in this article is, therefore: how well in fact do those adults do, judged as parents in the present, and not just as decision makers for the future?

We regard all the participants in care proceedings as sharing this parental responsibility (and, for brevity, we will refer to these participants collectively as 'the system' or 'the local authority and its partners'). This is because although day-to-day parental responsibility is exercised by the local authority, the local authority is required to follow the directions of-and answer for its actions to-the court, which, in turn, weighs up the views of the children's guardian (the courtappointed independent social worker, whose task is to represent the views and interests of the child), as well as those of the parents and the other parties and participants. For example, if a contact arrangement is ordered against the recommendation of the local authority, and if this turns out to be harmful to the child, then it would not be reasonable to hold the local authority alone responsible.

\section{Methodology and its limitations}

For a fuller account of the methodology, see McKeigue and Beckett (2009). In the area covered by Local Authority X, fifty-nine children from thirty-eight families were made subject to care proceedings during the financial year 2004-05, and these children were the focus of the study. (Since we followed cases through to their conclusion, the study was ongoing well into 2006.) The local authority notified the authors of all relevant cases and information was gathered by interviewing the allocated social workers involved from the outset of proceedings until the final hearing, at approximately three-month intervals. Interviews were guided by a detailed proforma, which asked for factual information, as well as opinions, on a variety of issues. At the analysis stage, we first extracted purely factual and quantitative data and then carried out a form of 'qualitative thematic analysis' (Seale, 2004, p. 314).

Since we had no access to files, we were reliant on the social workers for information. We talked only to local authority social workers and heard nothing, except indirectly, of the views of other participants. (The implications of this are discussed more fully in the previous article.) In particular, we did not solicit the views of the children themselves. Since the mean age of the children was four years two months, and children under one year old constituted 29 per cent of the total (strikingly close to the figure of 28.5 per cent of children in this age group identified in Masson et al.'s national study (2008)), it would not in any case have been practicable to solicit the views of children in a meaningful sense from a substantial part of the sample. There would also be considerable ethical issues involved in subjecting children involved in care proceedings to yet more questions from yet more strangers. Although it would be very interesting to hear the views of older children with experience of this process, our own study does not provide such a perspective. Another limitation of the methodology is that it does not allow us to comment in detail on the court process.

\section{About the in-court period}


In the Introduction, we identified three stages for children involved in care proceedings: precourt, in-court and post-court. In all three of these stages, a child may be in several different kinds of placement. Pre-court, a child will most typically be in the care of one or both of her own parents. Forty-seven per cent of our children in our sample were at home at the time of the first care hearing, with a further 12 per cent having been at home until removed to fostercare on an EPO some time within the previous month. However, about 15 per cent of the children in our sample were in the care of a relative, and 25 per cent were in local authority foster-care on a voluntary basis.

In the post-court period, there will be an agreed plan for the child's long-term future towards which the local authority will work. This could entail a return home, kinship care, long-term fostering or adoption. In our sample, the plan at the conclusion of proceedings was: placement with relatives, 22 per cent; return or remain at home, 22 per cent; adoption by nonrelatives, 34 per cent; long-term fostering, 17 per cent. There were two children for whom more than one possible plan was still open at that point.

During the in-court period (see Table 1), a child may likewise be in a variety of placements. What marks out this period is not the nature of the placement, but the status of the child. By definition, while the case is before a court, a child's long-term future has not yet been decided. The child is on a voyage in which he or she cannot know the destination, or how long it will take to reach it. As already emphasised, this experience will form a significant part of childhood, likely to contribute significantly in its own right (regardless of what happens before or afterwards) to the long-term development of that child, because it will most likely have taken place at a formative stage, and because it will almost inevitably have been an emotionally difficult time (Beckett, 2001). The uncertainty of the situation is, by its very nature, emotionally challenging. The way that the process is managed can add to this insecurity by placing additional emotional burdens on the child.

\begin{tabular}{|l|c|c|}
\hline Table 1. Placement during proceedings \\
\hline Type of placement & $\begin{array}{l}\text { Number of children }(\mathrm{n}=59) \\
\text { spending part of proceedings in } \\
\text { a placement of this kind }\end{array}$ & $\begin{array}{l}\text { Number of children }(\mathrm{n}=59) \\
\text { spending entire proceedings in a } \\
\text { single placement of this kind }\end{array}$ \\
\hline Foster-care & $42(71 \%)$ & $24(41 \%)$ \\
\hline Kinship care & $16(27 \%)$ & $11(19 \%)$ \\
\hline Parental home & $16(27 \%)$ & $3(5 \%)$ \\
\hline Residential children's home & $1(2 \%)$ & 0 \\
\hline $\begin{array}{l}\text { Parent-and-child residential } \\
\text { assessment unit }\end{array}$ & $4(7 \%)$ & 0 \\
\hline
\end{tabular}

\section{Findings: the in-court period in Local Authority $X$}

The remainder of this article looks at fifty-nine children for whom care proceedings were initiated in Local Authority X during the period 1 April 2004 to 31 March 2005 and discuss how well they were 'parented' by those responsible for their care during that period. We should make clear that when we do identify short-comings; these tend to be systemic and are rarely to be laid at the door of individuals.

\section{Long waits}

A good parent would not keep a child in a state of anxious uncertainty for longer than was absolutely necessary. The longer the wait for a decision in care proceedings, the longer the child has to endure the anxiety of having no long-term security. And too long a wait can reduce the likelihood of security ever being achieved, as Harriet Ward and her colleagues found in their study of forty-two babies in public care:

The longer children waited for a permanent placement, the more likely they were to experience further change and instability, and the more their chances of developing secure relationships were jeopardised (Ward et al.,2006, p. 125). 
The mean duration of proceedings experienced by the Local Authority $X$ children was thirtyseven weeks, within the target figure of forty weeks set by the government in the then Protocol (Department for Constitutional Affairs, 2003), which has since been superseded by the Public Law Outline (Ministry of Justice, 2008). The shortest set of care proceedings we encountered was nine weeks, the longest 103 weeks (just under two years). The forty-week limit set by the protocol was exceeded in 14 per cent of cases. Local Authority $\mathrm{X}$ and its partners look to have been doing substantially better in this regard than was typical nationally, if we may use for comparison Masson et al.'s (2008) national study of care applications made in 2004 (as opposed to financial year 2004-05 in our case). In 51.7 per cent of their sample, the duration of proceedings exceeded forty weeks. The average figure of thirty-seven weeks is an encouraging finding, but the reader is reminded that the existence of both pre- and postcourt delay, in addition to in-court delay, might considerably increase the period of uncertainty as subjectively experienced by the child.

Statistics also conceal individual stories. Many of these cases do still give cause for concern about the duration of the process if we look at them individually. The child in Case $\mathrm{JJ}$, for instance, was a few weeks old at the start of proceedings, which continued for fifty-three weeks. This child's whole babyhood - the time during which children naturally begin to form strong attachments-therefore passed in a temporary care placement. In Case N, the child concerned was just over two at the outset of proceedings, which lasted sixty-nine weeks, so, by the end, he had spent nearly 40 per cent of his life thus far subject to care proceedings and in temporary care. Case G involved a child aged two months at the outset and lasted Caring for Children during Care Proceedings Page 5 of 16103 weeks, a week less than two years (though the child was returned to her mother's full-time care after eighty-eight weeks while the proceedings were still continuing).

In the case of four children, the time spent waiting for a decision from a court was also not their first such wait. The child in Case $\mathrm{H}$ was made subject to proceedings at the age of nearly eleven, which lasted sixty weeks, itself some 10 per cent of her life to date. But this period was part of a much longer history, for, at the time proceedings were initiated, she was living with relatives with whom she had been placed on a 'permanent' basis as a result of a previous set of proceedings. Case $\mathrm{Y}$, involving a boy who was ten and a half at the outset, lasted thirty-four weeks, well within the Protocol target, but these were the second set of proceedings he had been through, and he had experienced multiple moves between carers over a long period prior to the first set of proceedings, adding up to a very long period in which he must have had little sense of permanence. Case $L$ involved two children aged eight and four in proceedings lasting fifty-seven weeks. A further move was required in order to implement the agreed care plan at the end. Previous proceedings that lasted eleven months had finished eighteen months previously. These children had spent over two years of their lives subject to care proceedings, half of the life of the younger children, a quarter of the life of the older child.

Even in cases in which there are no previous proceedings and no pre-court delay, completing a case in less than forty weeks should not be cause for complacency. Forty weeks is, under any circumstances, a long time for a child to have to wait for a decision. Even a case that is completed in thirty weeks, therefore, may still be regarded as having been delayed if an equally thorough assessment of the situation could feasibly have been achieved in a shorter timeframe. We encountered several instances of cases delayed for reasons to do with the limitations of the system, rather than to do with the needs of the child, such as court timetable problems, delays in completing assessments and reports, non-availability of experts or children's guardians.

\section{Stability}

'Stability' is one of the dimensions that social workers are required to use to assess parenting capacity (Department of Health, 2000, p. 21) and it is appropriate to use it as a benchmark against which to measure the parenting performance of Local Authority $X$ and its partners. A good parent tries to provide a manageable routine for a child, without too much change to cope with, and with continuity of care. Typically, during care proceedings, children have to cope with a number of changes. All the children in our study, with the exception of three, who 
remained at home throughout, had at some point to leave one home and live elsewhere. For the majority, care proceedings meant being looked after by people they had not known before. (Only 24 per cent lived with members of the extended family or with a parent for the whole duration of the proceedings.) And even if these placements were stable throughout the proceedings, the relationships formed with carers had to be temporary investments and the majority of the cohort were in fact to move to another home with different sets of carers at the conclusion of proceedings. Fifty-six per cent of them definitely would have to move again, and there were another 5 per cent whose current carers were still being assessed as long-term carers. (This compares less favourably with the Masson et al. (2008) sample, of which only 32.5 per cent were not yet in their permanent placement before the final hearing. It is likely, therefore, that the relatively short duration of care proceedings in Local Authority $\mathrm{X}$, as compared to the Masson et al. sample, will be offset by more post-court delay.)

Multiple placement moves are recognised to be harmful to children and they may result in a child developing long-term difficulties with attachment: '...multiple breaks can lead to the child being virtually unable to make true relationships' (Jones et al., 1991, p. 118). In our previous study in a different local authority (Beckett and McKeigue, 2003), we encountered several instances where children endured multiple placement moves in the course of care proceedings. For example, 'Bill George', aged fifteen months at the start of proceedings:

...had already been accommodated three times with three different sets of fosterparents... and was with the third set when the interim care order was made. $\mathrm{He}$ [and his newborn sibling]...were then moved successively to their mother, to another family member, to a new set of foster-parents, back to the foster-parents Bill was with at the onset of proceedings and on to yet another new set of foster-parents. They were later placed with adopters (Beckett and McKeigue, 2003, pp. 33-4).

Our view remains that imposing this amount of instability on children who were already in an inherently difficult and insecure situation amounts to 'system abuse' of children, a form of child abuse (see Beckett, 2007, pp. 196-200), and, as we have already noted, it seems likely to us that such children will sustain long-term emotional damage from their experiences, as other authors have also argued (Goldstein et al., 1984; Steinhauer, 1991).

It was therefore heartening to report that in the year in question, Local Authority $\mathrm{X}$ was able to avoid subjecting any children to the number of placement moves experienced by 'Bill' and others in our previous study. The average number of placements during proceedings was 1.4 and the maximum number was three. (These figures are based on a 'placement' being a person or set of people by whom a child is cared for during the period between the initial and final hearing during proceedings. Thus, if the child was with his own parents for part of the proceedings, then this was classed as a placement. If the child was moved, with his parent, to a residential assessment unit, this was not classed as a separate placement from being at home with the parent. Short respite care stays away from the main placement were excluded from our count-and were, in any case, quite rare.) If we exclude placements with the child's own parents, the average number of placements during proceedings, was 1.1. Five per cent of children were never removed from home throughout the proceedings, and 19 per cent were in the same kinship placement throughout the proceedings (see Table 1). Masson et al.'s (2008) much larger national study found that children were not moved during proceedings in only 17.6 per cent of cases, while in 8.4 per cent of cases, children had four moves or more, so Local Authority X's performance again looks to be above average.

However, as noted before, averages hide individual cases. There were three instances in which children were moved during the course of proceedings in circumstances which, from the child's perspective, would probably feel like rejection. (We draw attention to these because it seems to us this is something to avoid if possible, but of course we acknowledge that these problems could not necessarily have been predicted by Local Authority $\mathrm{X}$ and its partners. Even good parents have bad luck.) It is worth noting that all three cases involved kinship carers - a reminder that 'blood is thicker than water' is not necessarily a valid assumption. There is still a good deal that we need to learn about the relative advantages of kinship and foster-care, as Dunne and Kettler (2006) observe. Hunt et al. (2008) found a 
slightly higher disruption rate for children placed with kin than for those placed with strangers, though they urge caution in interpreting these figures. Chamberlain et al. (2006), in a US study, found the opposite. We can only observe here that both researchers found themselves wondering at times, when kinship care had been chosen, whether the choice had always been made for the right reasons.

In Case $\mathrm{H}$, the child, then aged nine, had already been subjected to emotional and physical abuse by the kinship carers with whom she had been placed following a previous set of proceedings. During the current sixty-week in-court period, she had to be moved from one foster-home to another because the foster-parents were having difficulty in coping with her behaviour.

In Case $\mathrm{K}$, three children had been moved, prior to the start of proceedings, from a mother and step-father who had physically abused them and subjected them to deliberately cruel and humiliating treatment. They had been taken to stay with a father who had, from the outset, expressed some reluctance to have them. During proceedings, the father felt unable to care for the middle child (aged eight) and took her to an aunt, who later also felt unable to care for her, with the result that the child had to be moved yet again to a foster-home.

The child in Case D was initially placed with an aunt and uncle following removal from her own parents. The social worker's feeling, three months into this arrangement, was that the uncle had no affection for the child and, in fact, disliked her. Three months further on, the child was moved to local authority foster-care. At the end of proceedings, this new placement, too, was under strain, the social worker describing the carers as being 'resentful' of the child, and indicating that she might need to be moved again to another temporary placement before being placed (as was the final plan) for adoption. One wonders about the prospects for the adoptive placement itself. By the time she was placed, this girl could be eight years old and outcome studies suggest that the breakdown rate for adoptions made at this age may be something in the order of 20 per cent (Performance and Innovation Unit, 2000).

\section{Disruption of childhood}

Even if placements remain stable, many other adults come and go in the lives of children subject to care proceedings. We were particularly concerned by the large numbers of staff involved in the supervision and transport arrangements for family contact and parenting assessments. Such changes of personnel would mean that the emotional challenge of going from placement to family of origin and back again would be exacerbated for the child, not just once, but repeatedly, by the fact that the adult in charge is not a trusted and familiar figure, but a stranger. The lack of continuity was in many instances further exacerbated by the use of multiple venues. Case D, for example, involved a girl of nearly seven at the outset who had two placements during the period of the care proceedings. She had contact with her mother three times a week. On Saturdays, this took place at her grandmother's house, on Tuesdays, it happened alternately at one of two different family centres in two different towns, and on Thursdays, it took place at yet another family centre. The supervision and transport on Saturdays were provided by the extended family, on Tuesdays by an independent agency regularly employed by the local authority for this purpose. (For brevity, we will call it the 'Star' agency. It was often not clear either to us or to our interviewees how many workers from the agency were involved in each case.) On Thursdays, supervision and transport were carried out either by the social worker or by one of two different unqualified support workers. If we discount the Saturday contact, which took place in a venue and with people she already knew, this child had to cope with three different supervision venues and at least four different supervisors.

Case $C$ involved two children of five and nearly four years. Contact with their mother took place initially in the offices of a voluntary organisation and was supervised by either the social worker or one of two unqualified support workers. For a period, contact was moved to a different venue, with another social worker and another support worker taking over the supervision. The Star agency also became involved in providing transport to and from the contact sessions. The social worker commented that the Star agency involved many different people in this work, over and above the five different workers from the local authority already 
taking part in contact supervision. In the final interview, the social worker told us that she had been very worried about the numbers of people 'going in and out of the children's lives' and the effect this was having on the children. 
The following are some other examples in brief. Case $\mathrm{H}$ (child aged eight at outset) involved supervision and transport being provided by either the social worker, a family support worker, local authority family centre staff or staff from the Star agency. Case I (five children aged from birth to twelve) involved supervision by Star agency staff, the social worker or one of several family support workers. Case R involved the social worker, family support worker or staff from the Star agency. Case $\mathrm{J}$ involved staff from the Star agency or a family support worker. Case BB (the child was aged four at outset) involved contact taking place at two different offices supervised by either the social worker, one of two different family support workers or staff from a local authority unit specialising in family assessment.

Apart from the sheer numbers of people and places involved, it is also worth considering the amount of time that assessment and contact visits can take up. In Case II, for example, a baby who had sustained several non-accidental injuries at the age of three months was taken to an Assessment and Contact Centre for three hours three days per week and one and a half hours for another two days per week, transported there and back over a period of several months by staff from the Star agency. The child was simultaneously involved in an assessment of parenting capacity of her parents by a psychologist. This assessment took place over a couple of months, involving the child in assessments of both parents together and of them separately with her. It also meant travel to and from the clinical setting in which the assessment took place, which was situated at some distance from the foster-home.

In Case V, the child, who was just a few days old when removed from parental care, had, by order of the court, daily contact with her mother at a contact centre. The child had to be woken for this. According to the social worker, the child was sufficiently unsettled by this that foster-carers were unable to get her into a routine until the contact was stopped at the end of the court process, whereupon she almost immediately became much more settled. In Case K, the children had twice-a-week contact with her mother at various venues, and once-a-week contact with their father at another venue. The children were also involved directly in three different assessments. In Case I, the children had contact with their mother three times a week in three different sibling combinations. In Case N, the children had contact with their mother three times a week, and were also directly involved in two separate parenting assessments and two other assessments. In Case W, the children had three-times-a-week contact with their mother and once-weekly contact with their father.

In some cases, the frequency of contact also seemed out of kilter with the quality of the relationship that the contact was supposed to be maintaining. In Case $\mathrm{P}$, in the middle of proceedings, the children aged two and one, had contact with their mother for two hours three times a week at a family centre, after two of which sessions they would have an additional hour with their grandmother. They also had contact with their father once a week. These children had been subject to quite severe neglect, including being deprived of food: the older child would physically shake when given food or would offer it to his sister. The social worker said that he would go straight for the toys on contacts with his mother, and was indiscriminate about which adults he went to. Clearly, this is important information for assessment purposes, but one wonders about the effect on children of being sent back again and again, for several hours several times a week, into a relationship from which they have been removed because of serious maltreatment. In Case $\mathrm{H}$, the child, aged ten at the outset, had contact with her brother and former kinship carers (against one of whom there were significant allegations of quite calculated emotional cruelty and physical abuse) three times a week. In Case F, a girl of five had repeatedly expressed to the social worker her wish not to have to have contact with her stepfather whose alleged physical abuse of her had led to the proceedings but, despite this, contact was ordered for the mother and stepfather together.

High levels of contact, which nearly always occurred in cases where the child was very young, or where the parents were separated and needed separate contact arrangements, or in cases where contact with grandparents and other extended family members had been allowed, could lead to small children's routines being disrupted and older children being subjected to a schedule that must have eaten into the time available for normal socialising, play and relaxation. Dame Butler-Sloss famously cautioned that 'the child is a person and not an object of concern' (Butler-Sloss, 1987, p. 245). If we lose sight of the fact that the in-court 
period is part of a childhood, there seems to us to be a danger of objectifying children in just this way, as they are ferried to and fro, day after day, between a bewildering variety of adults, with a bewildering variety of agendas and in a bewildering variety of locations.

\section{Changes of social worker}

In addition to contact supervisors, children also had to get used to the children's guardian and often to psychologists or psychiatrists called as expert witnesses (though, in many cases, these experts' involvement was limited to one-off contact). Against this background, one might hope that the local authority social worker who co-ordinates these various events in the child's life would be a source of stability here, but social workers themselves were frequently among the people coming and going. More than half of the children in our sample experienced at least one change of social worker during the proceedings, with additional changes at the beginning and end. It is worth noting that interviewing looked-after children aged ten to seventeen, Munro found that high turnover was the biggest source of complaints about social workers (Munro, 2000, p. 131).

Compared to the children in Masson et al.'s (2008, p. 30) national study, Local Authority X seemed to be doing relatively well. In the national study, 21.9 per cent of children had between three and five social workers during proceedings, but, in our sample, no child had more than three social workers during proceedings. Eight per cent had three, 47 per cent had two and 44 per cent had just one throughout. These figures, however, do not include changes before and after. It was normal practice in Local Authority $X$ to change social workers immediately before the outset of proceedings and again at the end of proceedings, due to these different stages being managed by different organisational units.

In Case D, the child had been through two placements during proceedings and it looked, at the end of our involvement, as if she might have to move again to another set of fosterparents before being placed with adopters. (We discussed earlier the very complex contact arrangements for this child, with four venues and many supervisors.) In this context, it was not surprising to hear that she was unhappy when she found out that she was also going to have yet another change of social worker at the end of proceedings, the present one having taken over from her predecessor at the beginning. The social worker commented that the child kept asking why this change was necessary. Asked at the end of the proceedings about how the experience had been for the child, the social worker commented: 'She's found it hard to know who to trust'; 'I don't think she's felt very loved over the past few months'; 'It's been a difficult and confusing time for her'. The word 'confusion' was frequently used by social workers to describe the impact of proceedings on children.

Discussing their own concerns about changes of social worker, Ward and her colleagues observe that 'secure and enduring relationships with social workers...might, perhaps, compensate for lack of stability in other areas of their lives' (Ward et al., 2008, p. 167). Case $D$, it seems to us, illustrates both this need, and the failure of the system to meet it.

\section{Uncertainty as to who is in charge}

We would suggest that good parents will try to convey that they are confident and in control, in order to make a child feel safe in their care. But we were given a wide range of answers by the social workers to the question: 'Who is driving the process?' The judge and the lawyers were those most frequently cited, with local authority managers and guardians next and very few seeing themselves as key players. The feelings of powerlessness expressed by social workers were sometimes quite striking, as was their lack of ownership of the process. In Case $\mathrm{KK}$, for instance, the social worker admitted she did not know the details of the different assessments that were underway or what these assessments would entail for the participating children.

We also had some evidence to suggest that changes of social worker were sometimes a factor here, since, on several occasions, we were told by social workers that they did not know the answer to a question because it related to events that occurred before their involvement. This resonates with an observation by Ward et al., based on their study of 478 
looked after children, that 'there was some indication that the more social workers allocated to any child, the less accurate or complete was the information on the case file' (Ward et al., 2008 , p. 45). It seems to us that if the social workers, who are assumed to be the link between the court system and the child or family, are not able to explain what is going on and why, children, parents and their carers will almost inevitably be unclear as to what is happening and why.

\section{Discussion and conclusions}

Good parents give their children 'a sense of being specially valued' (Department of Health, 2000 , p. 21). Our subjective impression was that social workers in Local Authority $X$ were in general trying hard to achieve this. Many children were also described as thriving in their temporary placements, suggesting that they were feeling cared for by their carers, too.

More objective measures allow us to assert that Local Authority $\mathrm{X}$ and its partners performed well against government targets for minimising the length of the period of uncertainty that is represented by the in-court period, although this was not true for all children in the sample and the total period of uncertainty for many children was much longer if we take into account their pre-court and post-court experiences.

Local Authority $\mathrm{X}$ avoided multiple placement moves of the order described in Beckett and McKeigue (2003). There were moves during proceedings, though, including instances where children experienced what would have felt like rejection by carers, so that immediate shortterm sources of acute distress were added to the long-term uncertainty that is inherent in the process itself.

Many children were exposed to a bewildering array of adults, and were shuttled between many different places. We wondered whether adults were privileging their decision-making rituals over children's current needs. It seems to us that if children are to feel valued, they need to feel valued in the here and now. It is not sufficient to reassure them that their future needs are being taken seriously.

Changes of social worker during proceedings, while not as severe a problem in Local Authority $\mathrm{X}$ as it appears to be nationally, could not have helped children to feel secure and cared for, and it seemed to us unfortunate that this authority's organisational structure resulted in changes of social worker being the norm before and after proceedings.

The system also did not always seem to give a clear message as to who was in charge and what was happening. In some instances, we felt it must have been difficult for children to get a sense of who was committed enough, or in a strong enough position, to look after their interests. Social workers often did not seem to have an overview of the whole process. Our impression was that changes of social worker would also be likely to weaken a child's sense of the process as a whole being managed, under control and safe.

Local Authority $\mathrm{X}$ and its partners have, in several respects, comfortably exceeded our expectations in terms of keeping the uncertainty of the in-court period within bounds and ensuring stability for children while going through it. However, we have also identified a number of ways in which children are not always well parented during this period, at least judged by the standards that we would normally apply to parenting. In particular, it seems to us that the care proceedings process runs a real danger of making children feel like objects of concern, rather than cared-for human beings. We suggest the following are areas in which changes might be considered.

First, our present study does not allow us to comment on deficiencies in the organisation of the court process, but we strongly support continued efforts to cut the in-court period to the minimum. We hope that the Public Law Outline (Ministry of Justice, 2008) will help, though we fear that it may have the effect of increasing pre-court delay, which is potentially just as harmful to children as over-long proceedings (McKeigue and Beckett, 2009). It is important to bear down simultaneously on pre-court, in-court and post-court delay, rather than focus exclusively on in-court delay. 
Second, we question the benefit of a degree of specialisation that results in children's cases being passed several times from one unit to another within a local authority, since changes of social worker are hard for children and possibly, too, result in a reduced sense of ownership on the part of social workers. A personal relationship between social worker and the child and family seems to us central if the child is to be a person and not an object of concern.

Third, although children have contact with two kinds of social worker during proceedings (local authority social worker and children's guardian), both kinds tend to be focused on the assessment and decision-making process as to a child's needs in the future. We wonder whether there is a case for someone different to have overall responsibility for the management of the children's care, and the meeting of their day-to-day needs, in the present, during the court period.

Finally, concurrent planning (Monck et al., 2003) was little in evidence in our sample, but would seem a way forward in reducing the number of children who reach the end of this lengthy process with yet one more placement move still ahead of them.

\section{Acknowledgement}

Ethical approval for this project was obtained in January 2004 from the Joint Schools Research Ethics Group of Anglia Ruskin University (then Anglia Polytechnic University).

\section{References}

Beckett, C. (2001) 'Children who wait for courts', British Journal of Social Work, 31(2), pp. 317-24.

Beckett, C. (2007) Child Protection: An Introduction, 2nd edn, London, Sage.

Beckett, C. and McKeigue, B. (2003) 'Children in limbo: Cases where care proceedings have taken two years or more', Adoption and Fostering, 27(3), pp. 31-40.

Butler-Sloss, Lord Justice E. (1987) Report of the Inquiry into Child Abuse in Cleveland, London, HMSO.

Chamberlain, P., Price, J., Reid, J., Landsverk, J., Fisher, P. and Stoolmiller, M. (2006) 'Who disrupts from placement in foster and kinship care?', Child Abuse and Neglect, 30(4), pp. 409-24.

Department for Constitutional Affairs (2003) Protocol for Judicial Case Management in Public Law Children Act Cases, London, Department for Constitutional Affairs.

Department of Health (2000) Framework for the Assessment of Children in Need and their Families, London, TSO.

Dunne, E. and Kettler, L. (2006) 'Social and emotional issues of children in kinship foster care and stressors on kinship carers', Children Australia, 31(2), pp. 22-9.

Goldstein, J., Freud, A. and Solnit, A. (1984) Beyond the Best Interests of the Child, new edn, London, Free Press.

Hunt, J., Waterhouse, S. and Lutman, E. (2008) 'Keeping them in the family: Outcomes for abused and neglected children placed with family or friends carers through care proceedings', Research Brief DCSF-RBX-05-08, March 2008, Department for Children Schools and Families.

Jones, D., Bentovim, A., Cameron, H., Vizard, E. and Wolkinel, S. (1991) 'Significant harm in context: The child psychiatrist's contribution', in Adcock, M., White, R. and Hollows, A. (eds), Significant Harm: Its Management and Outcome, Croydon, Significant Publications. Caring for Children during Care Proceedings Page 15 of 16

Masson, J., Pearce, J., Bader, K., Joyner, O., Marsden, J. and Westlake, D. (2008) Care Profiling Study, Ministry of Justice Research Series 4/08, London, Ministry of Justice.

McKeigue, B. and Beckett, C. (2009) 'Squeezing the toothpaste tube: Will reducing court delay lead to more pre-court delay?', British Journal of Social Work Advance Access, $10.1093 / \mathrm{bjsw} / \mathrm{bcn} 119$.

Ministry of Justice (2008) Public Law Outline: Guide to Case Management in Public Law Proceedings, London, Ministry of Justice. 
Monck, E., Reynolds, J. and Wigfall, V. (2003) The Role of Concurrent Planning, London, BAAF.

Munro, E. (2000) 'Empowering looked-after children', Child and Family Social Work, 6(2), pp. 129-37.

Performance and Innovation Unit (2000) Prime Minister's Review on Adoption, London, Cabinet Office.

Seale, C. (2004) 'Coding and analysing data', in C. Seale (ed.), Researching Society and Culture, 2nd edn, London, Sage, pp. 305-23.

Steinhauer, P. (1991) The Least Detrimental Alternative: A Systematic Guide to Case Planning and Decision-Making for Children in Care, Toronto, University of Toronto Press.

Ward, H., Holmes, L. and Soper, J. (2008) Costs and Consequences of Placing Children in Care, London, Jessica Kingsley.

Ward, H., Munro, E. and Dearden, C. (2006) Babies and Young Children in Care: Life Pathways, Decision-Making and Practice, London, Jessica Kingsley. Page 16 of 16 Chris Beckett and Bridget McKeigue 